Article

\title{
Synthesis and Characterization of Novel Pyridine Periodic Mesoporous Organosilicas and Its Catalytic Activity in the Knoevenagel Condensation Reaction
}

\author{
Fatemeh Rajabi ${ }^{1, *(\mathbb{D}}$, Arezoo Zare Ebrahimi ${ }^{1}$, Ahmad Rabiee ${ }^{2}{ }^{(\mathbb{D}}$, Antonio Pineda $^{3}$ and \\ Rafael Luque ${ }^{3}$ (D) \\ 1 Department of Science, Payame Noor University, P.O. Box: 19395-4697, Tehran 19569, Iran; \\ arezou.ebrahimi68@gmail.com \\ 2 Iran Polymer and Petrochemical Institute (IPPI), P.O. Box 112/14975, Tehran 19569, Iran; a.rabbii@ippi.ac.ir \\ 3 Department of Organic Chemistry, University of Cordoba, Ed. Marie Curie (C 3), Campus of Rabanales, \\ Ctra Nnal IV-A, Km 396, E14014 Cordoba, Spain; q82pipia@uco.es (A.P.); q62alsor@uco.es (R.L.) \\ * Correspondence: f_rajabi@pnu.ac.ir
}

Received: 20 January 2020; Accepted: 18 February 2020; Published: 2 March 2020

check for updates

\begin{abstract}
The preparation of novel organic-inorganic hybrid mesoporous organosilica containing pyridinedicarboxamide functional groups uniformly distributed inside the nanostructured pore walls has been addressed. The mesoporosity and uniformity of the synthesized nanomaterials were characterized by different techniques such as nitrogen adsorption/desorption measurements and powder X-ray diffraction (PXRD). Additionally, the presence of the pyridinedicarboxamide in the pore walls of the nanomaterials was assessed by Fourier-transform infrared spectroscopy (FT-IR), as well as ${ }^{29} \mathrm{Si}$ and ${ }^{13} \mathrm{C}$ solid-state cross-polarization and magic angle spinning nuclear magnetic resonance (CP/MAS-NMR). The Knoevenagel condensation of aldehydes with active methylene compounds was carried out over the pyridinedicarboxamide functionalized mesoporous organosilica, which has been proven to be an efficient heterogeneous basic catalyst in the presence of ethanol as solvent. The catalytic activity of the investigated materials was investigated in the Knoevenagel condensation between malononitrile and several benzaldehyde derivatives exhibiting a high conversion $(>90 \%)$ and excellent selectivity toward the final condensation products under very mild reaction conditions. Furthermore, the catalyst stability is noteworthy as it could be recycled and reused at least twelve times without any significant change in the performance.
\end{abstract}

Keywords: periodic mesoporous organosilica; pyridine carboxamide; base catalysts; Knoevenagel condensation

\section{Introduction}

The design of mesoporous functionalized silicate catalysts has been targeted since the development of the M41S silicate materials. Many alternatives have been proposed for the introduction of functionalities in mesoporous silicates. Among such options to load active sites on inert silicates, these can be classified as direct or "in situ" methodologies and post-synthetic approaches [1]. Among these last strategies, grafting [2], wet impregnation [3], or other most recently reported approaches such as ball-milling [4] can be included. Alternatively, the "in situ" methodologies go through the addition of the functional group directly into the sol-gel mixture. Thus, silicates can be modified "in situ" with metals, including zirconium and aluminum, among others, as well as organic functionalities. In this sense, periodic mesoporous organosilicas (PMOs) represent a class of hybrid nanomaterials with the presence of organic bridging groups incorporated into the highly ordered silica frameworks via covalent Si-C bonds [5-7]. These materials are very attractive because of the 
combination of a stable inorganic support together with the possibility of material functionalization with a wide range of organic moieties homogeneously distributed among the solid structure. PMOs, owing to the possibility of incorporating different functionalities into their walls, may have a potential use in a wide variety of applications such as catalysis [8-10], gas adsorption [11,12], decontamination [13], optical purposes [14], microelectronics [15], and drug delivery [16].

The common preparation method for PMO materials involves hydrolysis and the subsequent polycondensation of polysilsesquioxane, a bis-silylated organosilica precursor, around a template. After aging and formation of the organosilica, the template is removed and a porous structure of PMO material is obtained. Although the bridge of the silane is a key factor, there are many other factors that can affect PMO preparation including template, swelling agents, additives, catalyst, $\mathrm{pH}$, temperature, reaction time, and stirring rate [6]. Various classical organic bridging groups have been widely used including very simple units such as methane, ethane, ethene, benzene, and so on, in addition to alternative organic moieties, such as bridging molecules containing heterocyclic and chiral molecules. The organic function in the silica bridge will determine the chemical and physical properties of the PMO material such as a hydrophobic or hydrophilic surface, hydrothermal and mechanical stability, chemical reactivity, and the potential use of the materials.

Regarding catalytic applications, the number of reactions catalyzed by acidic PMOs reported is much higher than the number of reactions assisted by the basic PMOs catalysts. For instance, a basic urea-derived framework was used as the catalyst for $\mathrm{CO}_{2}$ coupling with epoxides [17]. Further, the use of amine bridges PMO materials has been reported as a catalyst for several reactions catalyzed by basic sites such as the Knoevenagel reaction or the solvent-free Henry reaction [18]. More recently, imine bridged PMOs were synthesized and successfully employed in the Knoevenagel condensation between aldehydes and ethyl cyanoacetate in water [19].

In this work, we report the incorporation of a pyridine dicarboxylate as bridging groups uniformly distributed into the silica framework as basic PMO material, developing in this way a crystal-like PMO catalyst containing heterocyclic pyridine dicarboxylate groups from a newly designed 2,6-pyridinedicarboxamide triethoxysilane precursor in the presence of a nonionic surfactant template. Such pyridinedicarboxamide units aim to be densely and regularly incorporated with in the highly ordered structure of the pore walls. The catalytic performance of as-synthesised materials was investigated in the Knoevenagel condensation reaction between aromatic aldehydes and malononitrile using ethanol as a solvent.

\section{Results and Discussion}

Herein, novel PMO material bearing pyridinedicarboxamide bridging groups (PMO-Py), bis(3-(triethoxysilyl)propyl)pyridine-2,6-dicarboxamide (2), were synthesized according to a two-step approach described on Scheme 1. Firstly, the formation of diethyl pyridine-2,6-dicarboxylate takes place via an acid-catalyzed esterification of pyridine-2,6-dicarboxylic acid with ethanol. Subsequently, the ester (1) formed in the first step undergoes an amidation reaction catalyzed by sodium ethoxide using 3-(triethoxysilyl)propyl amine, as outlined in Scheme 1. Eventually, the PMO-Py material was then synthesized by hydrolysis and condensation of in aqueous acidic solution in the presence of nonionic triblock copolymer surfactant P123 as a structure directing agent (SDA).

The efficient incorporation of pyridinedicarboxamide moieties into the material structure was confirmed by Fourier-transform infrared spectroscopy (FT-IR), which is depicted on Figure 1. In this sense, the presence of both silica framework as well as the organic moieties was confirmed. Firstly, the characteristic bands at $1628 \mathrm{~cm}^{-1}$ and $1595 \mathrm{~cm}^{-1}$ corresponding to the $\mathrm{C}=\mathrm{N}$ stretching vibration and $C=C$ stretching vibration of the pyridine ring, respectively, can be observed. In addition, the carbonyl group $(\mathrm{C}=\mathrm{O})$ stretching vibration band was detected at $1685 \mathrm{~cm}^{-1}$, which may confirm the presence of the amide in the as-synthesised material. Furthermore, the amide occurrence in the material can be confirmed by the presence of the N-H stretching vibration band $\left(3284 \mathrm{~cm}^{-1}\right)$ together with the presence at $\sim 3051 \mathrm{~cm}^{-1}$ and $2923 \mathrm{~cm}^{-1}$ can be attributed to asymmetric and symmetric C-H 
stretching corresponding to the pyridine ring and the propyl chain, respectively. In addition, other bands observable on Figure 1 are the $C-C$ stretching vibration, which is found at $1486 \mathrm{~cm}^{-1} ; C-C, C-O$; and $\mathrm{C}-\mathrm{H}$ bending vibrations, which were found in the region between 500 and $1000 \mathrm{~cm}^{-1}$. Regarding to silica framework, the characteristic stretching vibrations of $\mathrm{Si}-\mathrm{O}-\mathrm{Si}$ frameworks at approximately 506,743 , and $1096 \mathrm{~cm}^{-1}$ can be clearly observed. The condensed silica network was reported to exhibit a wide absorption band at about $3145 \mathrm{~cm}^{-1}$, attributed to the $-\mathrm{OH}$ stretching vibrations of silanol groups. These results point out that the pyridinedicarboxamide group was successfully incorporated into the PMO framework.

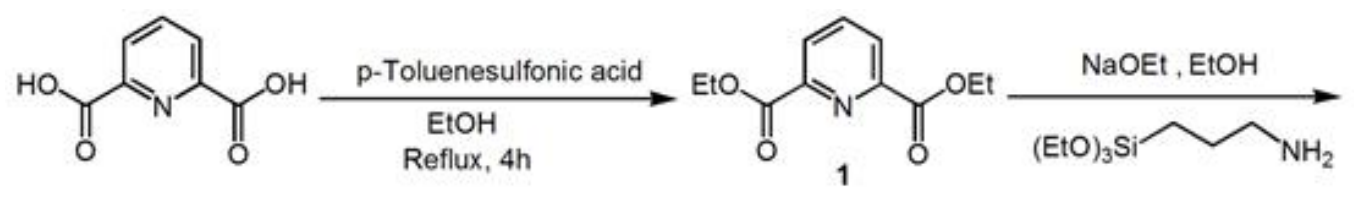

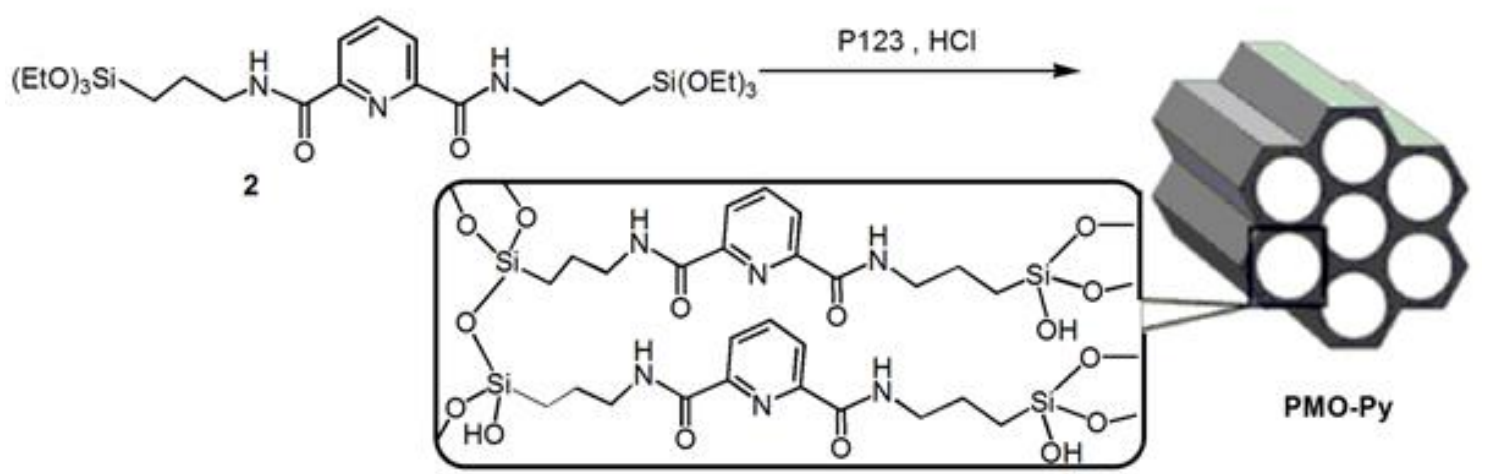

Scheme 1. Illustrative representation of the synthesis of pyridine loaded periodic mesoporous organosilica (PMO) materials.

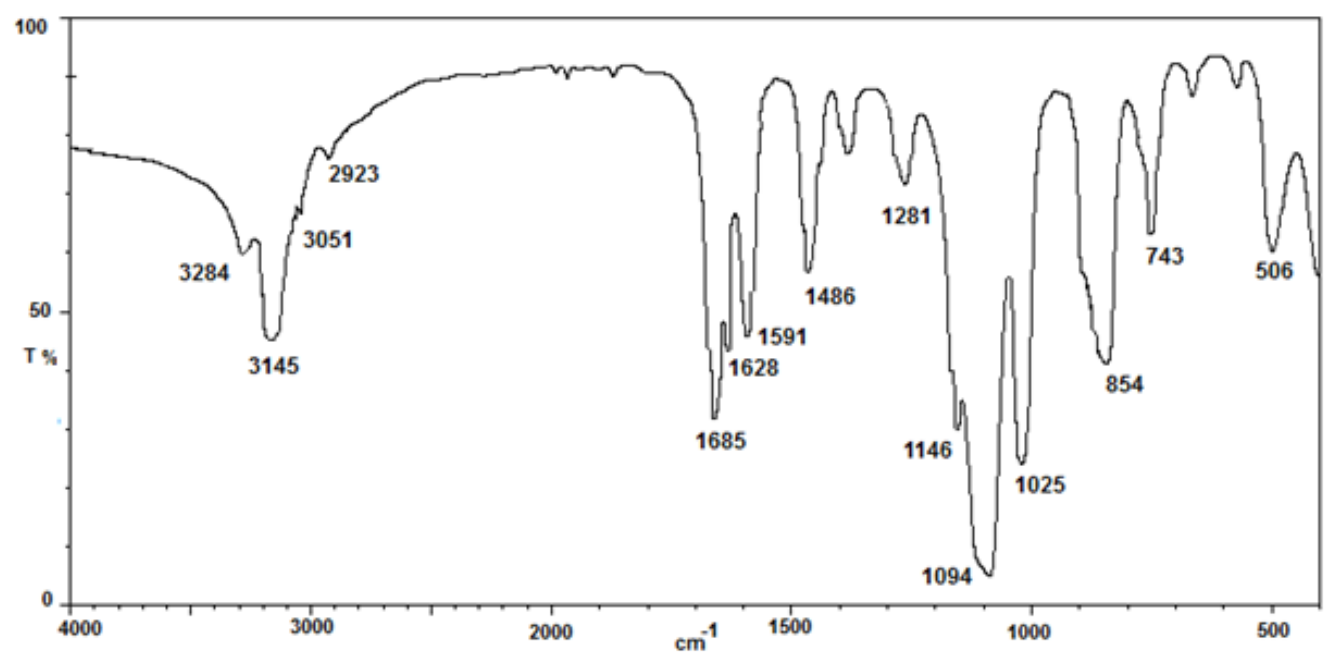

Figure 1. Fourier-transform infrared spectroscopy (FT-IR) spectrum for the periodic mesoporous organosilica $(\mathrm{PMO})$ material bearing pyridinedicarboxamide bridging groups.

Besides FT-IR analysis, solid-state ${ }^{29} \mathrm{Si}$ and ${ }^{13} \mathrm{C}$ nuclear magnetic resonance (NMR) (Figure 2) measurements were performed to obtain additional structural information and confirm the incorporation of pyridinedicarboxamide bridging groups in the material. Regarding the solid-state ${ }^{29} \mathrm{Si}$ NMR spectrum, there are signals with high intensity attributed to $\mathrm{T} 1$ species $\left(\mathrm{CSi}(\mathrm{OSi})(\mathrm{OH})_{2}\right)$ at $66 \mathrm{ppm}$, T2-silicon species $\left(\mathrm{CSi}(\mathrm{OH})(\mathrm{OSi})_{2}\right)$ at $-70.8 \mathrm{ppm}$, and $\mathrm{T} 3$ species $\left(\mathrm{CSi}(\mathrm{OSi})_{3}\right)$ at $-78.8 \mathrm{ppm}$ [20]. Then, it can be said that $\mathrm{Si}$ atoms are bound to an organic group and three $\mathrm{O}$ atoms $\left(\mathrm{R}-\mathrm{SiO}_{3}\right)$, which agrees with 
the expected structure in the material. Furthermore, the absence of any $\mathrm{Q}$ signals, which is because of the $\mathrm{Si}$ atoms linked to four $\mathrm{O}$ atoms [21], points out that the $\mathrm{Si}-\mathrm{C}$ bonds remain intact during the functionalization processes.

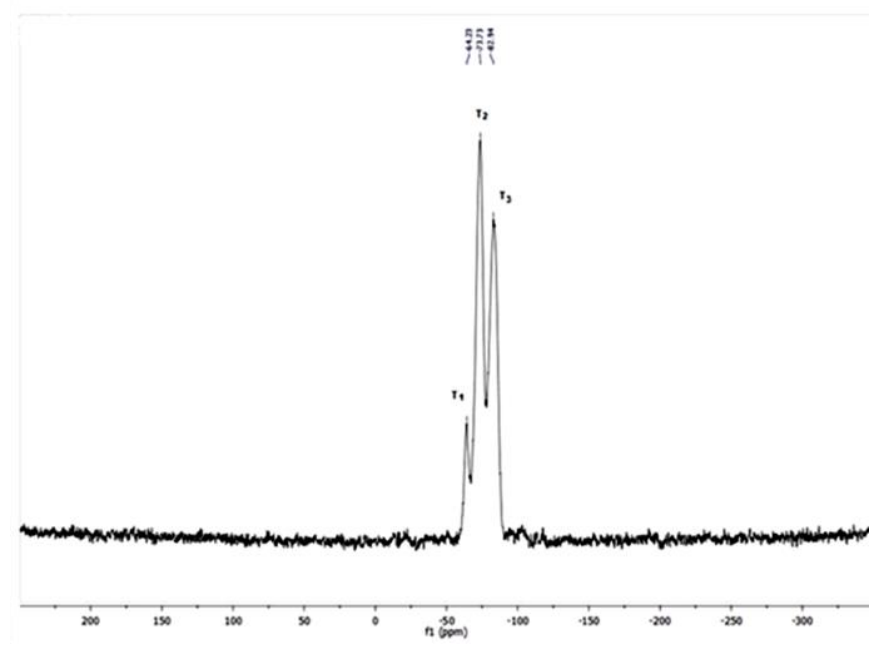

Figure 2. ${ }^{29} \mathrm{Si} \mathrm{CP} / \mathrm{MAS}$ nuclear magnetic resonance (NMR) spectrum of the pyridine periodic mesoporous organosilica (PMO-Py).

${ }^{13} \mathrm{C}$ cross-polarization and magic angle spinning (CP/MAS) NMR (Figure 3) measurements confirmed the successful incorporation of pyridinecarboxamide group into the PMO framework. The ${ }^{13} \mathrm{C} \mathrm{CP} / \mathrm{MAS}$ NMR spectrum of the pyridine ring-PMO shows a signal at $162.98 \mathrm{ppm}$ corresponding to the carbonyl group of the amide side chain. Moreover, the signals arising from the pyridine ring are clearly visible at 143.00, 134.16, and 127.36 ppm [22]. Additionally, several signals at a higher field can be assigned to the resonances of the propylene group at $62.22 \mathrm{ppm}\left(\mathrm{N}-\mathrm{CH}_{2}\right), 23.42 \mathrm{ppm}\left(-\mathrm{CH}_{2}-\right)$, and $7.04 \mathrm{ppm}\left(\mathrm{Si}-\mathrm{CH}_{2}\right)$ in the PMO-Py chain.

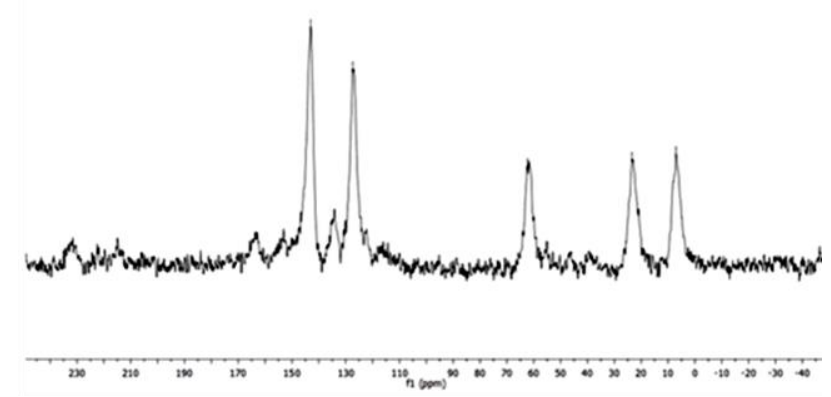

Figure $3 .{ }^{13} \mathrm{C} \mathrm{CP} / \mathrm{MAS}$ NMR spectrum of the PMO-Py material bearing pyridinedicarboxamide.

The characterization performed using thermogravimetric analysis/differential thermogravimetric analysis (TGA/DTGA) was employed to assess the thermal stability of PMO nanomaterials bearing pyridinedicarboxamide. Such an analysis was conducted from room temperature to $800{ }^{\circ} \mathrm{C}$ with a heating rate of $10^{\circ} \mathrm{C} / \mathrm{min}$ under static air atmosphere, and the respective TG and DTG curves of the PMO-Py are displayed in Figure 4. Firstly, a weight loss of approximately $9.7 \%$ owing to physisorbed water, ethanol, and loss of water by the condensation of residual silanol and ethoxy groups up can be observed up to $150{ }^{\circ} \mathrm{C}$. The following important weight loss (ca. 60.5\%) taking place between 500 and 
$650{ }^{\circ} \mathrm{C}$, as particularly visible in the DTG curve, is ascribed to the complete collapse of structure and thermal decomposition of the organic moieties. Therefore, it could be concluded that as-synthesised PMO-Py materials show a rather stable behavior towards temperature, keeping the framework of the PMO-Py material stable up to $500{ }^{\circ} \mathrm{C}$. Thus, further modifications could be carried out without a significant degradation of the mesoporous structure.

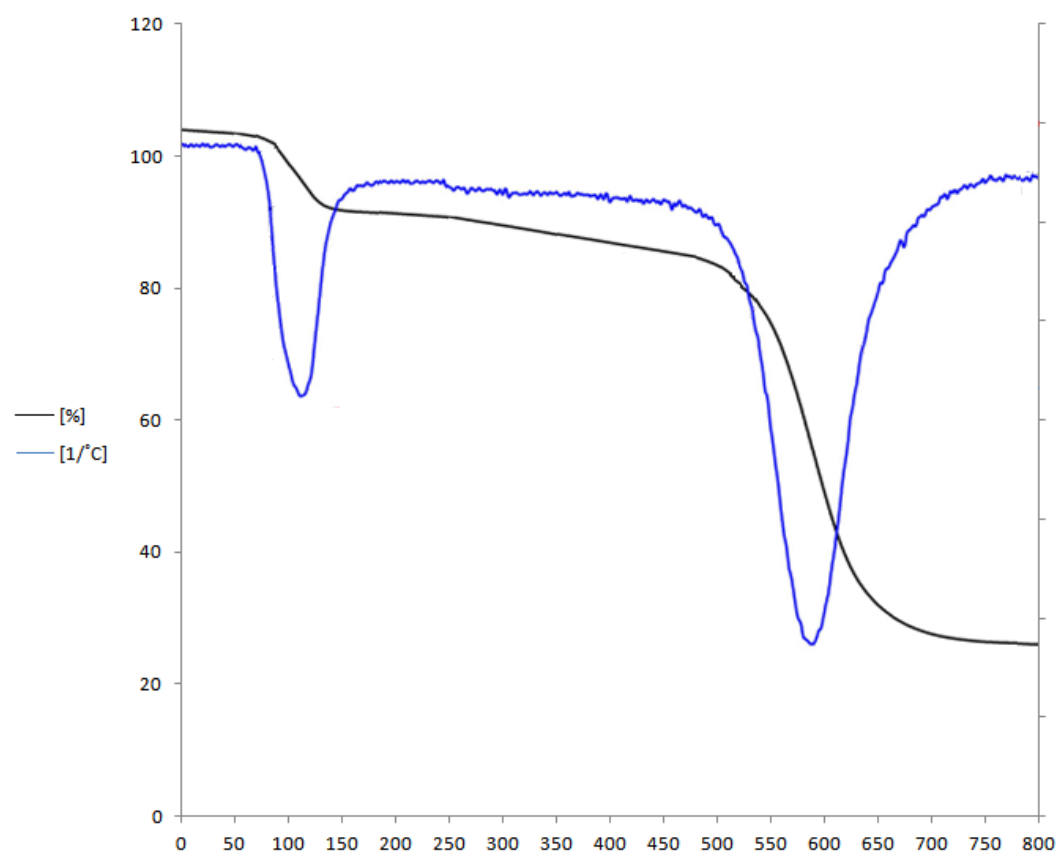

Figure 4. Thermogravimetric analysis/differential thermogravimetric (TGA/DTG) curves corresponding to the PMO-Py material up to $800^{\circ} \mathrm{C}$.

Nitrogen adsorption/desorption isotherm plots of the PMO-Py material (Figure 5) pointed to type IV isotherm curves, according to the IUPAC classification, characteristic of mesoporous materials. The synthesized materials displayed a high surface area of $616 \mathrm{~m}^{2} / \mathrm{g}$, as determined by the Brunauer-Emmet-Teller (BET) equation. In addition, the pore size distribution curve reveals a very narrow pore distribution centered at $7.5 \mathrm{~nm}$ (as can be seen in Figure S1) in the mesopore range, and confirms the ordered structure of the Py-PMO material. Finally, it is important to also highlight the high pore volume of this material $0.68 \mathrm{~cm}^{3} / \mathrm{g}$, calculated according to the Barrett, Joyner, and Halenda (BJH) method.

Regarding the structure of as-synthesised PMO-Py material, the low angle X-ray diffraction (XRD) pattern (Figure 6a) clearly reveals three signals; the first is a sharp signal corresponding to the (100) reflection and two other less intense signals assigned to the (110) and (200) second-order reflections are clearly visible. Thus, the PMO-Py material presents well ordered 2D-hexagonal corresponding to the P6 $\mathrm{mm}$ space group. In addition, wide-angle X-ray diffractograms (Figure $6 \mathrm{~b}$ ) did not show any crystal phase revealing an amorphous nature of the synthesized material.

In this material, in the presence of 2,6-diamide substitution in the pyridine ring, most of the pyridine units in the PMO structure (PMO-Py) are much less basic as compared with pyridine. In contrast, this substituted derivative of pyridine makes strong ligands in forming complexes with transition metal ion because of the chelate effect. Thus, this basicity in the PMO-Py material makes it a suitable catalyst for the Knoevenagel condensation reaction, which is one of the most important reactions and is very useful, and one that has been widely employed for the formation of carbon-carbon bonds in organic synthesis [23-25]. These condensation reactions occur between carbonyl compounds, either aldehydes or ketones, and compounds containing methylene activated groups. This reaction is widely employed as a carbon-carbon bond coupling reaction in the preparation of important 
intermediates in drug production by the pharmaceutical industry [26]. Therefore, the Knoevenagel reaction has been traditionally used for the evaluation of the catalytic activity of solid base catalysts including PMO materials [25].

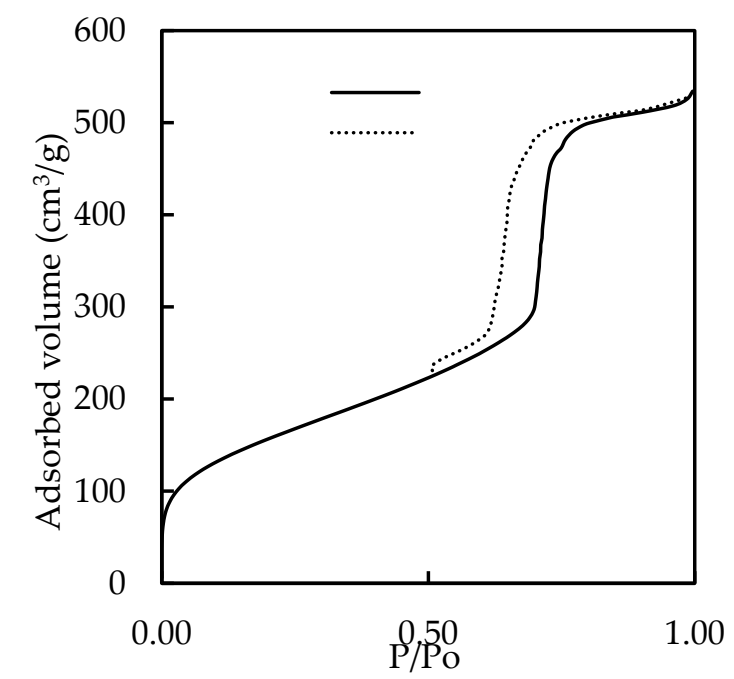

Figure 5. $\mathrm{N}_{2}$ adsorption/desorption isotherm plots for the Py-PMO material obtained at the liquid nitrogen temperature.
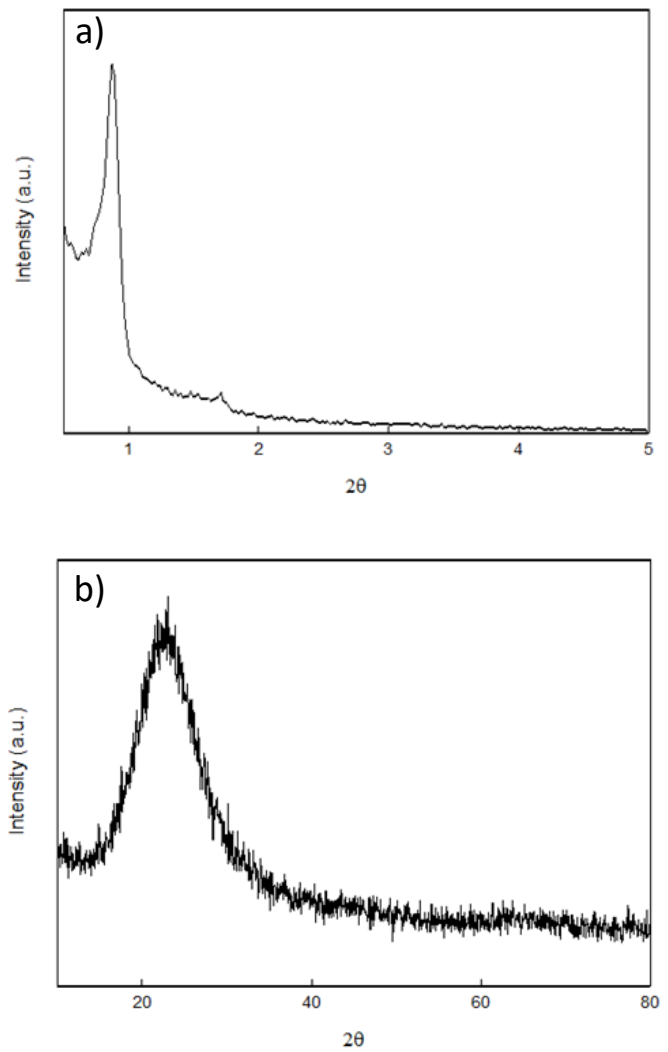

Figure 6. X-ray diffractograms corresponding to the PMO-Py material: (a) low-angle, (b) wide-angle.

The catalytic activity of PMO-Py was subsequently evaluated in the Knoevenagel condensation of aromatic aldehydes with an active methylene compound such as malononitrile. In this sense, the reaction conditions were optimized for $10 \mathrm{mmol}$ of malononitrile and $10 \mathrm{mmol}$ of benzaldehyde (also substituted compounds) using $20 \mathrm{mg}$ of PMO-Py as a catalyst using ethanol as solvent, according to Scheme 2. 


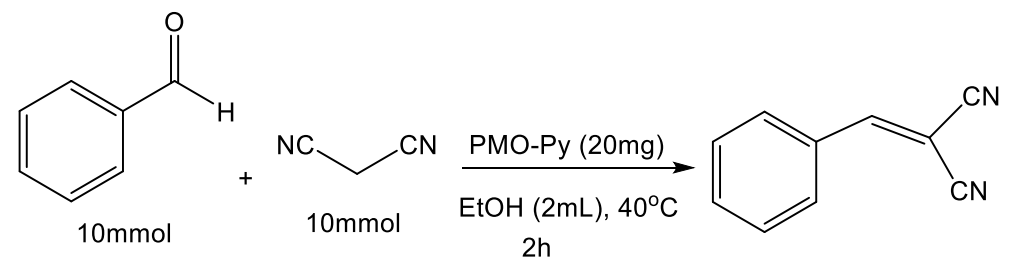

Scheme 2. Knoevenagel condensation between malononitrile and benzaldehyde derivatives catalyzed by PMO-Py.

Firstly, it is important to note that the PMO-Py material showed high conversion values for a relatively short reaction time under the investigated experimental conditions. There was not any additional product detected, so the selectivity was complete towards the $\mathrm{C}-\mathrm{C}$ coupling product. In addition, the catalytic material developed in this study was also investigated in the Knoevenagel reaction between other benzaldehyde derivatives, with malononitrile obtaining excelling catalytic activity results for these substrates as well. The differences found in the conversion for the different benzaldehyde derivatives were because of their electronic as well as steric effects (Table 1). As It has been previously reported $[27,28]$, the yield should be increased by the presence of electron withdrawing groups such as $-\mathrm{Cl}$ and $-\mathrm{NO}_{2}$. Nevertheless, this behavior it is not clear when PMO-Py is used as a catalyst after two hours, when the reaction is almost complete independently of the benzaldehyde used for the condensation with malononitrile. On the other side, when the substituents are electron releasing groups $\left(-\mathrm{OCH}_{3},-\mathrm{CH}_{3}\right)$, the conversion is slightly reduced as compared with the reaction using the non-substituted benzaldehyde. In addition, the effect of the use of different stereoisomers substituted benzaldehydes was investigated. In all of the cases, the use of the 4- regiomer (para-) led to the higher conversion as compared with the respective isomer substituted in 3- or 2-positions (orthoor meta-) owing to the steric hindrance effect.

Table 1. Knoevenagel condensation reaction of various aldehydes with malonitrile catalyzed by the periodic mesoporous organosilica (PMO)-Py catalyst.

\begin{tabular}{cccc}
\hline Entry & $\mathbf{R}$ & Conversion (\%) & Selectivity (\%) \\
\hline 1 & $\mathrm{H}$ & 99 & $>99$ \\
2 & $4-\mathrm{NO}_{2}$ & 98 & $>99$ \\
3 & $3-\mathrm{NO}_{2}$ & 95 & $>99$ \\
4 & $4-\mathrm{Cl}$ & 99 & $>99$ \\
5 & $2-\mathrm{Cl}$ & 95 & $>99$ \\
6 & $4-\mathrm{OMe}$ & 94 & $>99$ \\
8 & $2-\mathrm{Me}$ & 92 & $>99$ \\
9 & $4-\mathrm{Me}$ & 90 & $>99$ \\
10 & Blank & - & - \\
\hline
\end{tabular}

PMO-Py stability and recyclability in the Knoevenagel condensation reaction were also investigated. With this aim, the catalyst was isolated from the reaction mixture by filtration, washed with ethanol, and dried after each run for the following use in the Knoevenagel reaction. Thus, PMO-Py material exhibited a high stability without showing any significant catalytic activity loss after the 10th run in the condensation reaction between benzaldehyde and malononitrile under optimized conditions (Figure 7). 


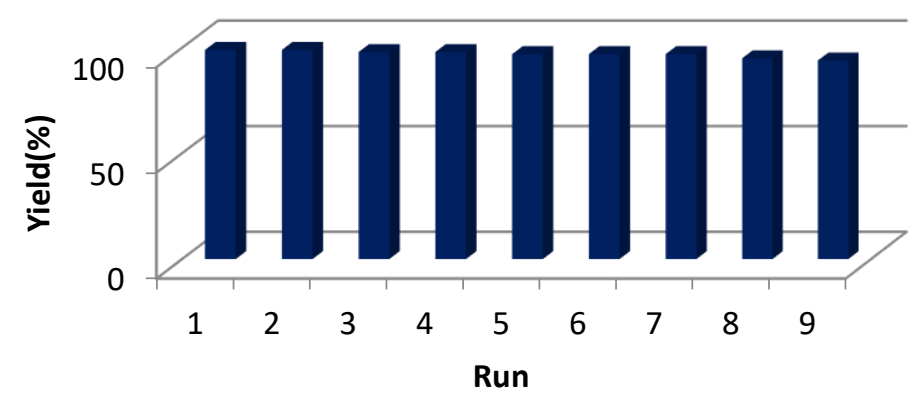

Figure 7. Reusability of the PMO-Py material in Knoevenagel condensation reaction between benzaldehyde and malononitrile under optimized conditions.

Table 2 show a comparison of the effectiveness of PMO-Py, with others reported in literature for the Knoevenagel reaction of benzaldehyde and malononitrile. The material herein synthesized according to a simple approach offers enhanced catalytic activity as compared with other basic catalytic materials investigated in the Knoevenagel reaction such as chitosan, a polysaccharide constitute of glucosamine units, which achieved analogous conversion as the PMO-Py material developed in this work after $4 \mathrm{~h}$ reaction, twice as high as the time required by the PMO-Py catalyst [28]. In addition, the conversion level achieved by the PMO-Py material improves the results achieved by other materials such as amino-functionalized mesoporous silicas [27], and is not far from others such as $\mathrm{MgO} / \mathrm{ZrO}$ [29].

Table 2. Comparison of the catalytic efficiency of PMO-Py in the reaction of benzaldehyde, malononirile and the previous literature.

\begin{tabular}{|c|c|c|c|c|c|}
\hline Entry & Catalyst & Condition & Time & Conversion (\%) & Ref. \\
\hline 1 & PMO-Py (20 mg) & PMO-Py & $2 \mathrm{~h}$ & 99 & This work \\
\hline 2 & amino-functionalized mesoporous silica (20 mg) & $\mathrm{EtOH} / \mathrm{RT}$ & $6 \mathrm{~h}$ & 90 & [27] \\
\hline 3 & Chitosan $(25 \mathrm{mg})$ & $\mathrm{EtOH} / 40{ }^{\circ} \mathrm{C}$ & $6 \mathrm{~h}$ & 99 & [28] \\
\hline 5 & PMO-IL-NTf2 (0.2 g) & $\mathrm{EtOH} / \mathrm{RT}$ & $20 \mathrm{~min}$ & 96 & [30] \\
\hline 6 & $\mathrm{SO}_{3} \mathrm{H}-\mathrm{AA} @ \mathrm{MNP}(20 \mathrm{mg})$ & $\mathrm{H}_{2} \mathrm{O}$-toluene $/ 90^{\circ} \mathrm{C}$ & $2 \mathrm{~h}$ & 94 & [31] \\
\hline 7 & Chol-SBA-15 (5mg) & $\mathrm{EtOH} / 60^{\circ} \mathrm{C}$ & $60 \mathrm{~min}$ & 100 & [32] \\
\hline
\end{tabular}

In addition to these, there are also relevant metal-encapsulated systems able to provide interesting results in knoevenagel chemistries as well as in biomedicinal applications $[33,34]$.

\section{Experimental}

\subsection{Synthesis of Diethyl Pyridine-2,6-Dicarboxylate}

Diethyl pyridine-2,6-dicarboxylate, employed as a bridging group, was prepared in a $250 \mathrm{~mL}$ round-bottom flask equipped with a magnetic stirring bar, which was loaded with $50 \mathrm{mmol}$ of pyridine-2,6-dicarboxylic acid and $1 \mathrm{mmol}$ of p-toluenesulfonic acid (p-TsOH) in $100 \mathrm{~mL}$ ethanol. Then, the reaction mixture was stirred and refluxed for $2 \mathrm{~h}$ and, eventually, upon reaction completion (followed by thin layer chromatography (TLC)), the solvent was removed in a rotary evaporator, obtaining a solid residue. The diethyl pyridine-2,6-dicarboxylate was obtained after dissolving the solid residue in about $30 \mathrm{~mL}$ of diethyl ether $\left(\mathrm{Et}_{2} \mathrm{O}\right)$, which was subsequently washed with 3-5 portions of $5 \% \mathrm{NaHCO}_{3}$ and water. Finally, the organic phase was dried over $\mathrm{MgSO}_{4}$, filtered, and the solvent was removed to obtain pure diethyl pyridine-2,6-dicarboxylate as a white solid with a $95 \%$ yield.

\subsection{Synthesis of Bis(3-(Triethoxysilyl)Propyl)Pyridine-2,6-Dicarboxamide}

A mixture of sodium ethoxide $(0.1 \mathrm{mmol})$, diethyl pyridine-2,6-dicarboxylate $(10 \mathrm{mmol})$, and 3-(triethoxysilyl)propyl amine $\left(20.5 \mathrm{mmol}\right.$,) was heated together in a sealed tube at $170{ }^{\circ} \mathrm{C}$ 
under stirring under nitrogen atmosphere for $5 \mathrm{~h}$. After cooling down the reaction mixture, chloroform was poured into the reaction tube to extract the reaction product. Subsequently, the suspension was filtered and the solvent was removed under vacuum, obtaining pure bis(3-(triethoxysilyl)propyl)pyridine-2,6-dicarboxamide as a white solid in $92 \%$ yield. $\mathrm{C}_{34} \mathrm{H}_{67} \mathrm{~N}_{3} \mathrm{O}_{11} \mathrm{Si}_{3}$ (778.17): the calculated composition was C: $52.48, \mathrm{H}: 8.68, \mathrm{~N}: 5.40$, while the actual one was C: $53.01, \mathrm{H}$ : 8.24, N: 5.14 .

\subsection{Synthesis of PMO Materials Bearing Pyridinedicarboxamide (PMO-Py)}

In a typical synthesis, $0.4 \mathrm{~g}$ of Pluronic P123 (Aldrich, average $\mathrm{Mw} \cong 5800$ ) was dissolved in $12.5 \mathrm{~g}$ of $2 \mathrm{M} \mathrm{HCl}$ solution with stirring at $35{ }^{\circ} \mathrm{C}$. Then, $2.3 \mathrm{~g}$ of bis(3-triethoxysilyl)propyl)pyridine-2,6-dicarboxamide was added and the resulting mixture solution was vigorously stirred at $35^{\circ} \mathrm{C}$ for $20 \mathrm{~h}$. The mixture was aged at $90^{\circ} \mathrm{C}$ overnight without stirring and, subsequently, the solid was filtered off and dried at $50^{\circ} \mathrm{C}$ overnight. The template was removed from the material through solvent extraction: $1.0 \mathrm{~g}$ of the as-synthesized sample was refluxed in a solution of $1.0 \mathrm{~mL} 37 \% \mathrm{HCl}$ and $100 \mathrm{~mL}$ ethanol for $12 \mathrm{~h}$, and then washed thoroughly with a mixture ethanol/water using a Soxhlet apparatus for $24 \mathrm{~h}$. Finally, it was dried in an oven at $80^{\circ} \mathrm{C}$ overnight.

\subsection{Material Characterisation}

Solid-state ${ }^{13} \mathrm{C}-\mathrm{NMR}$ cross-polarization (CP) and magic angle spinning (MAS) spectra were recorded in a Bruker $300 \mathrm{MHz}$ Ultrashield spectrometer (Rheinstetten, Germany). The sample spin rate was $8 \mathrm{kHz}$, proton length pulse was $2.5 \mu \mathrm{s}$, contact time was $2.4 \mathrm{~ms}$, and repetition time was $3 \mathrm{~s}$.

The textural properties of the synthesized material were measured by nitrogen physisorption measurements carried out at $77 \mathrm{~K}$ using a Micromeritics ASAP 2000 instrument (Micromeritics, Norcross, GA, USA). Samples were outgassed for $24 \mathrm{~h}$ at $10{ }^{\circ} \mathrm{C}$ under vacuum $\left(10^{-4} \mathrm{mbar}\right)$, and subsequently analyzed. Surface area was calculated using the BET (Brunauer-Emmet-Teller) equation, while the pore volume $\left(\mathrm{V}_{\mathrm{BJH}}\right)$ and pore size distributions $\left(\mathrm{D}_{\mathrm{BJH}}\right)$ were obtained the $\mathrm{BJH}$ method (Barrett, Joyner, and Halenda) in the $\mathrm{N}_{2}$ adsorption branch.

Powder X-ray diffraction patterns were recorded on a Bruker-AXS diffractometer using $\mathrm{Cu} \mathrm{K} \alpha$ radiation $(\lambda=1.5409 \AA)$ (Rheinstetten, Germany). Wide-angle diffraction pattern was recorded in the range $10^{\circ} \leq 2 \theta \leq 80^{\circ}$, with a step size of 0.017 and a scan time of $1.3 \mathrm{~s}$. Low-angle X-ray diffractogram was acquired in the range $0.5^{\circ} \leq 2 \theta \leq 5^{\circ}$ with $5 \mathrm{~s}$ time per step and a step size of $0.01^{\circ}$.

Thermogravimetric analysis (TGA) was performed from 25 to $800{ }^{\circ} \mathrm{C}$ using a heating rate of 10 ${ }^{\circ} \mathrm{C} \cdot \mathrm{min}^{-1}$ under static air atmosphere by a NETZSCH STA 409 PC/PG Instrument (Selb, Germany).

\subsection{Knoevenagel Reaction}

The catalytic tests of the as-synthesised material were performed in a $50 \mathrm{~mL}$ round-bottomed flask equipped with a magnetic stirring bar, which was charged with $10 \mathrm{mmol}$ aromatic aldehyde, $20 \mathrm{mg}$ of catalyst (PMO-Py), and $10 \mathrm{mmol}$ of malononitrile in $2 \mathrm{~mL}$ EtOH. The reaction mixture was stirred at $40^{\circ} \mathrm{C}$ for $2 \mathrm{~h}$. After that, the reaction mixture was filtered and the solid was washed with ethanol. The reaction was followed by withdrawing samples periodically that were subsequently analyzed by gas chromatography using an Agilent 6890N GC model equipped with a fitted with a DB-5 capillary column and an FID detector. To study the reusability of PMO-Py, the catalyst was separated after each cycle from the reaction mixture by simple filtration, washed with hot ethanol and dried at vacuum oven at $60^{\circ} \mathrm{C}$, and employed again in another reaction run.

\section{Conclusions}

A general and simple protocol was developed to synthesize periodic mesoporous organosilicas (PMOs) materials with bisilylated bridged pyridinedicarboxamide moieties uniformly distributed in the nanostructure pore walls. As-synthesized catalysts displayed a mesoporous nature with an arrangement typical for hexagonal ordered materials. The incorporation of pyridinedicarboxamide 
functionalities provided the as-prepared materials with the basic nature that was evaluated in the Knoevenagel condensation reaction.

The functionalized mesoporous organosilica proved to be a highly active, stable, and recyclable heterogeneous basic catalyst for the Knoevenagel condensation reactions between benzaldehyde and derivatives with malononitrile. Such pyridinedicarboxamide functionalized mesoporous organosilicas are expected to serve as a coordinated ligand to transition metals for further post-modifications and catalytic applications. Further studies on the preparation of catalysts containing transition metal are presently being carried out in our group.

Supplementary Materials: The following are available online at http:/www.mdpi.com/1996-1944/13/5/1097/s1, Figure S1: Pore size distribution curve corresponding to the material PMO-Py.

Author Contributions: A.Z.E. conducted all experimental work, F.R., A.R. and R.L. participated all aspect of this work. A.P. conducted formal analysis. All authors have read and agreed to the published version of the manuscript.

Funding: This research received no external funding.

Acknowledgments: F.R. would like to acknowledge Payame Noor University and Iran National Science Foundation (INSF) for support of this work. Antonio Pineda acknowledges the support of "Plan Propio de Investigación" from Universidad de Córdoba (Spain) and "Programa Operativo" FEDER funds from Junta de Andalucía. R.L. gratefully acknowledges funding from MINECO under project CTQ2016-78289-P.

Conflicts of Interest: "The authors declare no conflict of interest".

\section{References}

1. Vinu, A.; Hossain, K.Z.; Ariga, K. Recent Advances in Functionalization of Mesoporous Silica. J. Nanosci. Nanotechnol. 2005, 5, 347-371. [CrossRef]

2. Wang, X.; Lin, K.S.K.; Chan, J.C.C.; Cheng, S. Direct Synthesis and Catalytic Applications of Ordered Large Pore Aminopropyl-Functionalized SBA-15 Mesoporous Materials. J. Phys. Chem. B 2005, 109, 1763-1769. [CrossRef]

3. Campelo, J.M.; Luna, D.; Luque, R.; Marinas, J.M.; Romero, A.A. Sustainable Preparation of Supported Metal Nanoparticles and Their Applications in Catalysis. ChemSusChem 2009, 2, 18-45. [CrossRef]

4. Pineda, A.; Balu, A.M.; Campelo, J.M.; Romero, A.A.; Carmona, D.; Balas, F.; Santamaria, J.; Luque, R. A Dry Milling Approach for the Synthesis of Highly Active Nanoparticles Supported on Porous Materials. ChemSusChem 2011, 4, 1561-1565. [CrossRef] [PubMed]

5. Inagaki, S.; Guan, S.; Fukushima, Y.; Ohsuna, T.; Terasaki, O. Novel Mesoporous Materials with a Uniform Distribution of Organic Groups and Inorganic Oxide in Their Frameworks. J. Am. Chem. Soc. 1999, 121, 9611-9614. [CrossRef]

6. Melde, B.J.; Holland, B.T.; Blanford, C.F.; Stein, A. Mesoporous Sieves with Unified Hybrid Inorganic/Organic Frameworks. Chem. Mater. 1999, 11, 3302-3308. [CrossRef]

7. Asefa, T.; MacLachlan, M.J.; Coombs, N.; Ozin, G.A. Periodic mesoporous organosilicas with organic groups inside the channel walls. Nature 1999, 402, 867. [CrossRef]

8. Corma, A.; Das, D.; Garcia, H.; Leyva, A. A periodic mesoporous organosilica containing a carbapalladacycle complex as heterogeneous catalyst for Suzuki cross-coupling. J. Catal. 2005, 229, 322-331. [CrossRef]

9. Liu, J.; Yang, H.Q.; Kleitz, F.; Chen, Z.G.; Yang, T.Y.; Strounina, E.; Lu, G.Q.; Qiao, S.Z. Yolk-Shell Hybrid Materials with a Periodic Mesoporous Organosilica Shell: Ideal Nanoreactors for Selective Alcohol Oxidation. Adv. Funct. Mater. 2012, 22, 591-599. [CrossRef]

10. Liu, X.; Maegawa, Y.; Goto, Y.; Hara, K.; Inagaki, S. Heterogeneous Catalysis for Water Oxidation by an Iridium Complex Immobilized on Bipyridine-Periodic Mesoporous Organosilica. Angew. Chem. Int. Ed. 2016, 55, 7943-7947. [CrossRef] [PubMed]

11. Sim, K.; Lee, N.; Kim, J.; Cho, E.B.; Gunathilake, C.; Jaroniec, M. $\mathrm{CO}_{2}$ Adsorption on Amine-Functionalized Periodic Mesoporous Benzenesilicas. ACS Appl. Mater. Interfaces 2015, 7, 6792-6802. [CrossRef] [PubMed]

12. De Canck, E.; Ascoop, I.; Sayari, A.; Van Der Voort, P. Periodic mesoporous organosilicas functionalized with a wide variety of amines for $\mathrm{CO}_{2}$ adsorption. Phys. Chem. Chem. Phys. 2013, 15, 9792-9799. [CrossRef] [PubMed] 
13. White, B.J.; Zeinali, M. Photo-decontamination catalyst, useful for e.g., sample containing trinitrotoluene, comprises optically active molecule incorporated into periodic mesoporous organosilica. U.S. Patent US2007073095-A1, 29 March 2007.

14. Mizoshita, N.; Goto, Y.; Kapoor, M.P.; Shimada, T.; Tani, T.; Inagaki, S. Fluorescence Emission from 2,6-Naphthylene-Bridged Mesoporous Organosilicas with an Amorphous or Crystal-Like Framework. Chem. Eur. J. 2009, 15, 219-226. [CrossRef] [PubMed]

15. Mizoshita, N.; Ikai, M.; Tani, T.; Inagaki, S. Hole-Transporting Periodic Mesostructured Organosilica. J. Am. Chem. Soc. 2009, 131, 14225-14227. [CrossRef]

16. Teng, Z.; Zhang, J.; Li, W.; Zheng, Y.; Su, X.; Tang, Y.; Dang, M.; Tian, Y.; Yuwen, L.; Weng, L.; et al. Facile Synthesis of Yolk-Shell-Structured Triple-Hybridized Periodic Mesoporous Organosilica Nanoparticles for Biomedicine. Small 2016, 12, 3550-3558. [CrossRef]

17. Liu, M.S.; Lu, X.Y.; Shi, L.; Wang, F.X.; Sun, J.M. Periodic Mesoporous Organosilica with a Basic Urea-Derived Framework for Enhanced Carbon Dioxide Capture and Conversion Under Mild Conditions. ChemSusChem 2017, 6, 1010-1119. [CrossRef]

18. Zhu, F.; Yang, D.; Zhang, F. Amine-bridged periodic mesoporous organosilica nanospheres as an active and reusable solid base-catalyst for water-medium and solvent-free organic reactions. J. Mol. Catal. A 2012, 363-364, 387-397. [CrossRef]

19. Sun, Z.; Wang, Y.; Zhang, Z.; Zhu, F.; Zhao, P.; Li, G.; Shao, F.; Rui, J. Imine-bridged periodic mesoporous organosilica as stable high-activity catalytic for Knoevenagel reaction in aqueous medium. Res. Chem. Intermed. 2019, 45, 3107-3121. [CrossRef]

20. Yoshina-Ishiii, C.; Asefa, T.; Coombs, N.; MacLachlan, M.J.; Ozin, G.A. Periodic mesoporous organosilicas, PMOs: Fusion of organic and inorganic chemistry 'inside' the channel walls of hexagonal mesoporous silica. Chem. Commun. 1999, 2539-2540. [CrossRef]

21. Landskron, K.; Hatton, B.D.; Perovic, D.D.; Ozin, G.A. Periodic Mesoporous Organosilicas Containing Interconnected [Si(CH2)]3 Rings. Science 2003, 302, 266-269. [CrossRef]

22. Waki, M.; Inagaki, S. Periodic mesoporous organosilicas possessing molecularly mixed pyridine and benzene moieties in the frameworks. Micropor. Mesopor. Mater. 2019, 284, 10-15. [CrossRef]

23. Pineda, A.; Balu, A.M.; Campelo, J.M.; Romero, A.A.; Luque, R. Activity of amino-functionalised mesoporous solid bases in microwave-assisted condensation reactions. Catal. Commun. 2013, 33, 1-6. [CrossRef]

24. Schneider, E.M.; Zeltner, M.; Kränzlin, N.; Grass, R.N.; Stark, W.J. Base-free Knoevenagel condensation catalyzed by copper metal surfaces. Chem. Commun. 2015, 51, 10695-10698. [CrossRef] [PubMed]

25. Chughtai, A.H.; Ahmad, N.; Younus, H.A.; Laypkov, A.; Verpoort, F. Metal-organic frameworks: Versatile heterogeneous catalysts for efficient catalytic organic transformations. Chem. Soc. Rev. 2015, 44, 6804-6849. [CrossRef] [PubMed]

26. Walker, S.D.; Borths, C.J.; DiVirgilio, E.; Huang, L.; Liu, P.; Morrison, H.; Sugi, K.; Tanaka, M.; Woo, J.C.S.; Faul, M.M. Development of a Scalable Synthesis of a GPR40 Receptor Agonist. Org. Process Res. Dev. 2011, 15, 570-580. [CrossRef]

27. Mondal, J.; Modak, A.; Bhaumik, A. Highly efficient mesoporous base catalyzed Knoevenagel condensation of different aromatic aldehydes with malononitrile and subsequent noncatalytic Diels-Alder reactions. J. Mol. Catal. A 2011, 335, 236-241. [CrossRef]

28. Sakthivel, B.; Dhakshinamoorthy, A. Chitosan as a reusable solid base catalyst for Knoevenagel condensationreaction. J. Colloid Interface Sci. 2017, 485, 75-80. [CrossRef] [PubMed]

29. Gawande, M.B.; Jayaram, R.V. A novel catalyst for the Knoevenagel condensation of aldehydes with malononitrile and ethyl cyanoacetate under solvent free conditions. Catal. Commun. 2006, 7, 931-935. [CrossRef]

30. Yao, N.; Tan, J.; Liu, X.; Liu, Y.; Hu, Y.L. Multifunctional periodic mesoporous organosilica supported dual imidazolium ionic liquids as novel and efficient catalysts for heterogeneous Knoevenagel condensation. J. Saudi Chem. Soc. 2019, 23, 740-752. [CrossRef]

31. Wang, Z.; Yuan, X.; Cheng, Q.; Zhang, T.; Luo, Y. An efficient and recyclable acid-base bifunctional core-shell nanocatalyst for the one-pot deacetalization-Knoevenagel tandem reaction. New J. Chem. 2018, 42, 11610-11615. [CrossRef] 
32. del Hierro, I.; Pérez, Y.; Fajardo, M. Supported choline hydroxide (ionic liquid) on mesoporous silica as heterogeneous catalyst for Knoevenagel condensation reactions. Microporous Mesoporous Mater. 2018, 263, 173-180. [CrossRef]

33. Kankala, R.K.; Zhang, H.; Liu, C.-G.; Kanubaddi, K.R.; Lee, C.-H.; Wang, S.-B.; Cui, W.; Santos, H.; Lin, K.; Chen, A.-Z. Metal species-encapsulated mesoporous silica nanoparticles: Current advancements and latest breakthroughs. Adv. Funct. Mater. 2019, 29, 1902652. [CrossRef]

34. Kankala, R.K.; Liu, C.-G.; Yang, D.-Y.; Wang, S.-B.; Chen, A.-Z. Ultrasmall platinum nanoparticles enable deep tumor penetration and synergistic therapeutic abilities through free radical species-assisted catalysis to combat cancer multidrug resistance. Chem. Eng. J. 2020, 383, 123138. [CrossRef]

C 2020 by the authors. Licensee MDPI, Basel, Switzerland. This article is an open access article distributed under the terms and conditions of the Creative Commons Attribution (CC BY) license (http://creativecommons.org/licenses/by/4.0/). 